\title{
Zur Einberufung der Hauptversammlung durch die nach $\$ 122$ Abs. 3 AktG ermächtigte Aktionärsminderheit
}

\author{
Prof. Dr. Mathias Habersack, München \\ und \\ Prof. Dr. Peter O. Mülbert, Mainz*
}

Inhaltsübersicht

ZGR 2014, 1-23

I. Einführung $\ldots \ldots \ldots \ldots \ldots \ldots \ldots \ldots \ldots \ldots \ldots \ldots \ldots \ldots \ldots \ldots \ldots \ldots \ldots \ldots$

II. Grundlagen $\ldots \ldots \ldots \ldots \ldots \ldots \ldots \ldots \ldots \ldots \ldots \ldots \ldots \ldots \ldots$

1. Satzungsmäßige Anmeldung bei der Gesellschaft ( $(123 \mathrm{Abs} .2 \mathrm{AktG}) \ldots$

2. Einberufung durch den ermächtigten Aktionär $\ldots \ldots \ldots \ldots \ldots \ldots$

III. Befugnis des ermächtigten Aktionärs zur Benennung einer Anmeldestelle im

Besonderen. . . . . . . . . . . . . . . . . . . . . . 14

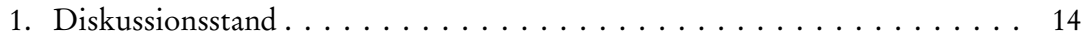

2. Geringe Aussagekraft des Wortlauts des $\$ 123$ Abs. 2 Satz 2 AktG . . . . . 15

3. Die Aktionärsbefugnis als Ausfluss der gesetzlichen Grundkonzeption . . . 16

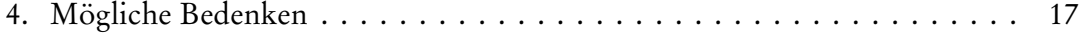

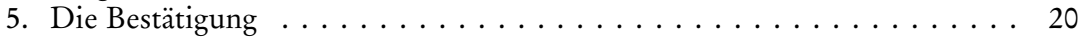

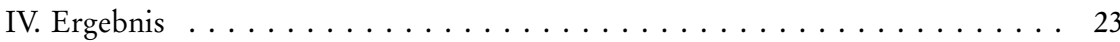

Die durch eine Aktionärsminderheit einberufene außerordentliche Hauptversammlung der Balda $A$ G vom 18. 7. $2013^{1}$ hat gezeigt, dass Rechtsstellung und Befugnisse der gerichtlich zur Einberufung ermächtigten Minderheit bislang wenig geklärt sind. In besonderer Weise gilt dies für die Frage, ob der einberufende Aktionär befugt ist, eine Anmeldestelle zu benennen, und in der Folge Aktionäre, die sich innerbalb der Frist des $\$ 123$ Abs. 3 Satz 2 Akt G unter der angegebenen Adresse anmelden, zur Teilnabme an der Hauptversammlung berechtigt sind. Der Beitrag bejabt diese Frage.

Under Section 122 Para. 3 of the German Stock Corporation Act, a court may authorise shareholders to call a shareholders' meeting, when a demand to call such a meeting had already been denied by the management board. Minority shareholders of the Balda AG,

* Prof. Dr. Habersack hat den Lehrstuhl für Bürgerliches Recht und Unternehmensrecht der Ludwig-Maximilians-Universität München inne; Prof. Dr. MüLberT ist Inhaber des Lehrstuhls für Bürgerliches Recht, Handels- und Wirtschaftsrecht, Bankrecht der Johannes Gutenberg-Universität Mainz und Direktor des Instituts für deutsches und internationales Recht des Spar-, Giro- und Kreditwesens an der Universität Mainz. Der Beitrag geht auf Anfragen aus der Praxis zurück.

1 Dazu AG Bad Oeynhausen, Beschluss vom 23. 8.2013, HRB 3077; ferner Börsen-Zeitung vom 19.7.2013, S. 11. 
pursuant to such authorisation, called an extraordinary shareholders' meeting for 18 July 2013. The events at the meeting demonstrated that minority shareholders' legal status and authority in such circumstances remain largely uncertain. In particular, if the articles of association require the shareholders to give notice of their attendance prior to the meeting, the German Stock Corporation Act is ambiguous as to whether a minority shareholder who calls a meeting may specify the mailing address to which shareholders have to give their notice. It is also unclear whether shareholders who give notice of their attendance to such an address are entitled to participate in the shareholders' meeting. This article analyses both issues and concludes that (a) a minority shareholder may specify the mailing address to which shareholders have to give their notice, and $(b)$ shareholders who give notice of their attendance to that address may participate in the shareholders' meeting.

\section{Einfübrung}

Macht die Satzung der $\mathrm{AG}^{2}$ in Ausübung der Ermächtigung des $₫ 123$ Abs. 2 Satz 1 AktG die Teilnahme an der Hauptversammlung von der vorherigen Anmeldung abhängig, bereitet das für den Regelfall der Einberufung der Hauptversammlung durch den Vorstand schon deshalb keine großen Schwierigkeiten, weil die enge Abstimmung des für die Einberufung und Vorbereitung der Hauptversammlung zuständigen Vorstands mit dem für die Legitimationsprüfung zuständigen Versammlungsleiter ${ }^{3}$ gewährleistet ist und der Vorstand durch Wahl der für die Anmeldung maßgebenden Adresse sicherstellen kann, rechtzeitig über die Anmeldeunterlagen verfügen zu können. Anderes gilt für die Einberufung durch eine Aktionärsminderheit gemäß $\$ 122$ Abs. 3 Satz 1 AktG. Bei ihr stellt sich bereits die Frage, wem die Vorbereitung der Hauptversammlung obliegt und welche Befugnisse insoweit bestehen; im engen Zusammenhang damit steht die Frage, ob die zur Einberufung ermächtigte Minderheit zur Wahl der Anmeldeadresse befugt ist. Beide Fragen lassen sich in Ermangelung klarer gesetzlicher Vorgaben nur unter Rückgriff auf allgemeine Grundsätze und Erwägungen beantworten.

Im Folgenden sind zunächst einige Grundlagen zum satzungsmäßigen Anmeldeerfordernis (unter II. 1.) und zur Einberufung einer Hauptversammlung durch den gerichtlich ermächtigten Minderheitsaktionär (unter II. 2.) zu entfalten. Hierauf aufbauend erfährt die zentrale Frage nach der Befugnis zur Benennung einer Anmeldestelle eine eindeutige Antwort dahin, dass in den Fällen des $\$ 122 \mathrm{Abs} .3 \mathrm{AktG}$ der Minderheitsaktionär hierzu allein berechtigt ist und keiner Genehmigung oder irgendwie gearteten Mitwirkung der Gesellschaft selbst bedarf (unter III.).

2 Der AG stehen die KGaA ( $\$ 278$ Abs. 3 AktG) und die SE (Art. 53 SE-VO) gleich.

3 Dazu unter II. 2. b). 


\section{Grundlagen}

\section{Satzungsmäßige Anmeldung bei der Gesellschaft (\123 Abs. 2 AktG)}

Das Erfordernis der Anmeldung bei der Gesellschaft beruht auf $\$ 123$ Abs. 2 AktG in der Fassung durch das UMAG aus dem Jahre 2005. Diese in sich geschlossene Regelung der satzungsmäßigen Anmeldung ${ }^{4}$ sieht in Satz 1 vor, dass die Satzung „die Teilnahme an der Hauptversammlung oder die Ausübung des Stimmrechts davon abhängig machen [kann], dass die Aktionäre sich vor der Versammlung anmelden“. Diese Anmeldung muss gemäß Satz 2 „der Gesellschaft unter der in der Einberufung hierfür mitgeteilten Adresse mindestens sechs Tage vor der Versammlung zugehen“. Entsprechendes gilt nach $\$ 123$ Abs. 3 Satz 3 für den Nachweis der Teilnahmeberechtigung.

Der bis zur Neufassung durch das UMAG geltende $\mathbb{1 2 3}$ AktG a. F. entsprach, was die Anmeldung zur Hauptversammlung anbelangt, sachlich weitgehend der heutigen Regelung. Sein Abs. 2 Satz 1 sah vor, dass die Satzung „die Teilnahme an der Hauptversammlung oder die Ausübung des Stimmrechts davon abhängig machen kann, ..., dass sich die Aktionäre vor der Versammlung anmelden“. Abs. 4 ergänzte dies dahingehend, dass es für die Anmeldung zur Versammlung genügt, wenn die Aktionäre „sich nicht später als am dritten Tag vor der Versammlung anmelden“.

Von der Anmeldung ist der Nachweis der Aktionärsstellung und der mit dieser verbundenen Berechtigung zur Teilnahme an der Hauptversammlung und zur Stimmrechtsausübung zu unterscheiden. Ein solcher Nachweis ist im Unterschied zum Anmeldeerfordernis obligatorisch, d.h. seine Einführung steht nicht im Belieben der Gesellschaft. Er ist für Namensaktien in $\$ 67 \mathrm{Abs.} 3$ AktG und für Inhaberaktien in $\ 123$ Abs. 3 AktG geregelt. $\ 123$ Abs. $3 \mathrm{AktG}$ verweist hinsichtlich des „Wie“ des Nachweises auf die Satzung und statuiert nur gewisse inhaltliche Vorgaben, innerhalb derer sich die Satzung bewegen muss.

\section{a) Ratio der Öffnungsvorschriften}

Über den Zweck der in $\$ 123$ AktG eröffneten Möglichkeit, in der Satzung die Anmeldung als zusätzliche Voraussetzung für die Teilnahme oder/und die Ausübung des Stimmrechts vorsehen, findet sich in der Begründung zu $\ 123$ AktG a. F. nichts. ${ }^{5}$ Demgegenüber heißt es in der Regierungsbegründung zum UMAG ausdrücklich:

4 Begr. RegE eines Gesetzes zur Unternehmensintegrität und Modernisierung des Anfechtungsrechts (UMAG), BT-Drs. 15/5092, S. 13.

5 S. Kropff, Aktiengesetz, 1965, S. $172 \mathrm{f} \mathrm{zu} \$ 123$ E-AktG a. F. 
„\$123 eröffnet ... der Satzung die Möglichkeit, Vorkehrungen und Anforderungen für die Anmeldung zu regeln. Dies ist bei börsennotierten Gesellschaften sachlich geboten und wird dort durchweg so gehandhabt.... Vor allem die börsennotierte Gesellschaft hat ein Interesse daran zu wissen, wie viele Aktionäre an der Hauptversammlung teilnehmen werden“" ${ }^{6}$

Das Schrifttum teilt dieses auf organisatorische Zwecke fokussierende Normzweckverständnis seit jeher nahezu einhellig. ${ }^{7}$ Erläuternd heißt es etwa bei Noack/Zetzsche:

„Die Gesellschaft soll wissen, mit wie vielen Teilnehmern zu rechnen ist, um angemessene Abwicklungssysteme, Räumlichkeiten, ggfs. Bewirtung und Personal bereitstellen zu können. Zudem ist das Teilnehmerverzeichnis ... vorzubereiten". 8

Baums formuliert mit leicht abweichender Akzentuierung wie folgt:

„Die Gesellschaft soll die Versammlungsorganisation auf die zu erwartenden Teilnehmer ausrichten können. Insbesondere ermöglicht die Anmeldung, die Eintrittskarten auszustellen und den Aktionären zuzuleiten sowie das Teilnehmerverzeichnis ... bereits auf der Grundlage der bei der Gesellschaft eingegangenen Informationen vorzubereiten und so den Ablauf der Hauptversammlung zu vereinfachen".9

In diesen Konkretisierungen spiegelt sich die wohl zum aktienrechtlichen Allgemeingut gehörende Erkenntnis, dass die Vorbereitung und Durchführung der Hauptversammlungen insbesondere von Publikumsgesellschaften hohe organisatorische und logistische Herausforderungen für deren jeweiligen Organisator mit sich bringen. ${ }^{10}$

\section{b) Zugang unter der in der Einberufung mitgeteilten Adresse}

Voraussetzung für eine wirksame Anmeldung ist, wie $\ 123$ Abs. 2 Satz 2 AktG nunmehr ausdrücklich formuliert, deren Zugang bei der Gesellschaft. Der Begriff „Zugang“ wird im Schrifttum teils i.S. v. \$130 BGB verstan-

6 Begr RegE UMAG, aaO (Fn. 4), S. 13.

7 S. nur GessLer, in: Geßler/Hefermehl/Eckardt/Kropff, AktG, Bd.2, 1972/74, $\mathbb{1 2 3}$ Rdn. 51; Werner, Großkomm. z. AktG, 4. Aufl., 1993-2008, $\$ 123$ Rdn. 55; Noack/ Zetzsche, Kölner Komm. z. AktG, 3. Aufl., 2011, \$123 Rdn.3; Hürfer, AktG, 10. Aufl., 2012, \$123 Rdn. 1 i. V. m. 6; Rieckers, in: Spindler/Stilz, AktG, 2. Aufl., 2010, $\$ 123$ Rdn. 8; Pluta, in: Heidel, Aktienrecht und Kapitalmarktrecht, 3. Aufl., 2011, \123 Rdn. 13; Drinhausen, in: Hölters, AktG, 2011, $\$ 123$ Rdn. 5; Herrler, in: Grigoleit, AktG, 2013, $\$ 123$ Rdn. 5; Baums, FS Hüffer, 2010, S. 15, 17; BAYER/Scholz/ WeIss, AG 2013, 742, 744.

8 Noack/Zetzsche, aaO (Fn. 7), $\$ 123$ AktG Rdn. 3.

9 Baums, FS Hüffer, S. 17.

10 Illustrativ hierzu die erfahrungsgesättigte Darstellung bei Butzke, Die Hauptversammlung der Aktiengesellschaft, 5. Aufl., 2011, Kap. B Rdn. 19 ff; ferner HöretH, in: Semler/Volhard/Reichert (Hrsg.), Arbeitshandbuch für die Hauptversammlung, 3. Aufl.,

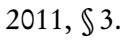


den. ${ }^{11}$ Unter Heranziehung der allgemeinen bürgerlich-rechtlichen Regeln zum Zugang unter Einschaltung dritter Personen - Empfangsboten und Empfangsbevollmächtigte - könnte der Zugang bei der Gesellschaft daher auch dadurch erfolgen, dass die Anmeldung bei einem Empfangsboten oder Empfangsbevollmächtigten der Gesellschaft zugeht. Indes verlangt Satz 2 zusätzlich den Zugang „unter der in der Einberufung hierfür mitgeteilten Adresse“. Der Zugang bei einem Empfangsboten oder Empfangsvertreter ist daher nur wirksam, wenn dieser in der Einberufung angegeben ist. Das gilt auch für den Fall, dass die Empfangsberechtigung - rechtlich ganz unbedenklich $^{12}$ - einem Hauptversammlungs-Dienstleister erteilt wird, der hierdurch als Anmeldestelle mit Boten- oder Empfangsvollmacht ${ }^{13}$ fungiert. Rechtlich unbedenklich umsetzen lässt sich das aus Satz 2 resultierende $\mathrm{Zu}-$ gangserfordernis mittels der - durchaus üblichen - Praxis, den eingeschalteten Dienstleister mittels Verwendung einer c/o-Adresse als empfangsberechtigte Anmeldestelle zu benennen. ${ }^{14}$

\section{c) Konzentrationswirkung der Adressmitteilung}

Der Angabe einer bestimmten Adresse in der Einberufung kommt nach h. M. eine Konzentrationswirkung dergestalt zu, dass ein wirksamer Zugang der Anmeldung ausschließlich unter der genannten Adresse möglich ist. ${ }^{15}$ Das soll nach h. M. auch im Falle der Einschaltung eines Empfangsvertreters oder Empfangsboten unter Verwendung einer c/o-Adresse gelten, so dass ein $\mathrm{Zu}-$ gang der Anmeldung am Ort der Hauptverwaltung oder der Geschäftsleitung

11 Noack/Zetzsche, aaO (Fn. 7), $\$ 123$ AktG Rdn. 104; Kubis, Münchener Komm. z. AktG, 3. Aufl., 2013, \123 Rdn.14; a.A. Ziemons, in: K. Schmidt/Lutter, AktG, 2. Aufl., 2010, $\$ 123 \mathrm{Rdn} .19$ (mit im hiesigen Kontext irrelevanten begründenden Überlegungen).

12 S. nur Noack/Zetzsche, aaO (Fn. 7), \$123 AktG Rdn. 107; Kubis, aaO (Fn. 11), \$123 AktG Rdn. 14; Butzke, aaO (Fn. 10), Kap. C Rdn. 41

13 Dass der derart eingeschaltete Dienstleister stets als Empfangsvertreter statt als Empfangsbote agiert (so etwa Noack/Zetzsche, aaO [Fn. 7], $\$ 123$ AktG Rdn. 107; Kubis, aaO (Fn. 11), $\$ 123$ AktG Rdn. 14), gebieten weder die Vorgaben des AktG noch die allgemeinen bürgerlich-rechtlichen Regeln (s. nur Ellenberger, in: Palandt, BGB, 73. Aufl., 2014, Einf. v. $\$ 164$ Rdn. 12).

14 OLG Frankfurt v. 20. 10.2009 - 5 U 22/09 (juris); LG Berlin BB 2009, 1265; NoACK/

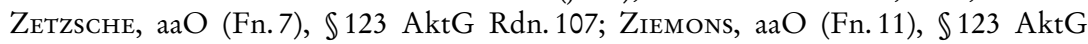
Rdn. 19.

15 Hüffer, aaO (Fn. 7), \123 AktG Rdn. 7; Noack/Zetzsche, aaO (Fn. 7), \123 AktG Rdn. 104 m. Fn. 126; Rieckers, aaO (Fn. 7), \$123 AktG Rdn. 16; Herrler, aaO (Fn. 7), $\$ 123$ AktG Rdn. 8; im Ausgangspunkt auch Ziemons, aaO (Fn.11), $\$ 123$ AktG Rdn. 18, die allerdings die Angabe der Postadresse stets für erforderlich hält, also auch bei Angabe einer oder mehrerer weiterer Zugangsadressen. 
der Gesellschaft keine wirksame Anmeldung im Sinne des $₫ 123$ Abs. 2 AktG bildet. ${ }^{16}$

Dass $\ 123$ Abs. 2 Satz 2 Halbsatz 1 . AktG eine Zugangskonzentrationsregel enthält, ist schon im Normtext angelegt, wenn es heißt: „Die Anmeldung muss der Gesellschaft unter der in der Einberufung hierfür mitgeteilten Adressen mindestens sechs Tage vor der Versammlung zugehen“. In dieser Formulierung kommt klar zum Ausdruck, dass der Mitteilung der Adresse in der Einberufung eine eigene rechtliche Bedeutung zukommt. Dem wird aber nur die Lesart als Konzentrationsregel gerecht, denn andernfalls wäre die Mitteilung einer Adresse in der Einberufung überflüssig, weil funktionslos. ${ }^{17}$ In teleologischer Hinsicht rechtfertigt sich diese Konzentrationsregel aus dem Zweck des $₫ 123$ Abs. 2 AktG, mit der Verfügbarmachung entsprechender Satzungsbestimmungen dem Einberufenden die organisatorische Vorbereitung der Hauptversammlung zu erleichtern. ${ }^{18}$ Denn die Verengung der Zugangsmöglichkeiten auf von dem Einberufenden festgelegte Zugangskanäle - oder gar nur auf einen Zugangskanal - vereinfacht insbesondere bei großen Gesellschaften die Organisation spürbar. Im Falle einer gesetzlichen $(\mathbb{\$} 16$ Abs. 4 WpÜG) oder statuarischen Verkürzung der Mindestfrist für die rechtzeitige Anmeldung gilt dies in erhöhtem Maße, wie im zulässigen Extremfall der statutarischen Verkürzung auf 0 Tage ${ }^{19}$ also dem Verzicht auf eine Anmeldefrist, überdeutlich wird.

\section{d) Zugang beim Feblen einer Adressmitteilung in der Einberufung}

Die Angabe einer besonderen Adresse für die Anmeldung bildet kein zwingendes Erfordernis für eine wirksame oder auch nur rechtmäßige Einberufung. Unterlässt die Gesellschaft versehentlich deren Angabe in der Einberufung oder verzichtet sie sogar bewusst hierauf, bildet dies weder einen Nichtigkeitsmangel (s. auch $₫ 241 \mathrm{Nr} .1 \mathrm{AktG}$ ) noch auch nur einen Anfechtungsgrund..$^{20}$ Vielmehr bewendet es für den Zugang bei den allgemeinen Vorschriften $(\$ 130$ BGB), so dass eine wirksame Anmeldung bei der Gesellschaft unter der Adresse des Ortes der Hauptverwaltung oder Geschäftsleitung zu erfolgen hat. ${ }^{21}$

16 Noack/Zetzsche, aaO (Fn. 7), $₫ 123$ AktG Rdn. 108; auch Hüffer, aaO (Fn. 7), $\$ 123$ AktG Rdn. 7; a. A. Kubis, aaO (Fn. 11), $\$ 123$ AktG Rdn. 14.

17 Zutreffend Noack/Zetzsche, aaO (Fn. 7), $\$ 123$ AktG Rdn. 104 in Fn. 126 a. E.

18 S. unter II. 1. a).

19 S. etwa Noack/Zetzsche, aaO (Fn. 7), $\$ 123$ AktG Rdn. 111; Kubis, aaO (Fn. 11), $\$ 123$ AktG Rdn. 13.

20 Noack/Zetzsche, aaO (Fn. 7), $\$ 123$ AktG Rdn. 108; Ziemons, aaO (Fn. 11), $\$ 123$ AktG Rdn. 18.

21 Hüffer, aaO (Fn. 7), \123 AktG Rdn. 7; Noack/Zetzsche, aaO (Fn. 7), \123 AktG Rdn. 108; Kubis, aaO (Fn. 11), $\$ 123$ AktG Rdn. 14; Ziemons, aaO (Fn. 11), $\$ 123$ AktG 


\section{e) Einseitige Dispositivität des Anmeldezugang-Erfordernisses}

Das Erfordernis des Zugangs der Anmeldung wird vom Schrifttum in der Sache für einseitig dispositiv erklärt, wenn es heißt: „Die Gesellschaft darf an falsche Adressen zugegangene Anmeldungen freiwillig berücksichtigen“".22 Diese lapidare Feststellung ist sachgerechte Konsequenz der ratio des $\mathbb{1 2 3}$ Abs. 2 AktG, mit der Verfügbarmachung entsprechender Satzungsbestimmungen die organisatorische Vorbereitung der Hauptversammlung zu erleichtern. ${ }^{23}$ Geht es nämlich darum, dem einberufenden Veranstalter der Versammlung die Vorgabe von Regularien zu erlauben, die diesem die Organisation der Hauptversammlung vereinfachen, dann ist es nur folgerichtig, dass dieser in bestimmten Grenzen auf deren Einhaltung auch verzichten kann.

Die Organzuständigkeit für den Verzicht der Gesellschaft auf den Zugang der Anmeldung liegt beim Versammlungsleiter. Dieser kann den Verzicht ausdrücklich erklären, aber auch konkludent, insbesondere durch die Einbeziehung der zwar nicht wirksam zugegangenen, wohl aber tatsächlich eingegangenen Anmeldungen in die Legitimationsprüfung. Hierbei hat der Versammlungsleiter materiell den Gleichbehandlungsgrundsatz ( $\$ 53$ a AktG) zu wahren und deshalb alle Aktionäre zu berücksichtigen, die sich, wenn auch unter falscher Adresse, angemeldet haben. Ein Verzicht auf die Wahrung des Zugangserfordernisses kommt daher nur in Betracht, wenn in der Folge alle nur tatsächlich eingegangenen Anmeldungen auch Berücksichtigung finden, nicht aber, wenn der Versammlungsleiter Anhaltspunkte dafür hat, dass ihm nicht alle diese Anmeldungen vorliegen.

\section{Einberufung durch den ermächtigten Aktionär}

\section{a) Grundsatz}

Die Einberufung der Hauptversammlung ist nach $\$ 121$ Abs. 2 Satz 1 AktG grundsätzlich Sache des Vorstands. Dieser hat die Hauptversammlung nicht

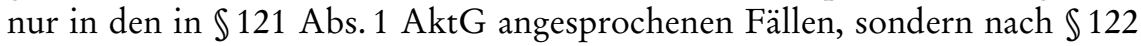
Abs. 1 Satz 1 AktG auch dann einzuberufen, wenn Aktionäre, deren Anteile zusammen den zwanzigsten Teil des Grundkapitals erreichen, die Einberufung schriftlich unter Angabe des Zwecks und der Gründe verlangen. $\$ 122$ Abs. 2 AktG ergänzt das Einberufungsrecht der Minderheit um das Recht auf Ergänzung der Tagesordnung einer anderweitig einberufenen Hauptver-

Rdn. 18; Rieckers, aaO (Fn. 7), $\$ 123$ AktG Rdn. 16; Drinhausen, aaO (Fn. 7), $\$ 123$ AktG Rdn. 8; Herrler, aaO (Fn. 7), $\$ 123$ AktG Rdn. 8; Butzke, aaO (Fn. 10), Kap. C Rdn. 41.

22 Noack/Zetzsche, aaO (Fn. 7), $\$ 123$ AktG Rdn. 104.

23 S. unter II. 1. a). 
sammlung. Die Vorschriften des $\ 122$ Abs. 1, 2 AktG bezwecken ersichtlich und unbestrittenermaßen den Schutz der Aktionärsminderheit, indem sie es dieser ermöglichen soll, ihre versammlungsgebundenen Rechte auszuüben. ${ }^{24}$ Mag auch das Ergebnis der Beschlussfassung offen sein, so erreicht die Minderheit mit Ausübung ihrer Rechte aus $\$ 122$ Abs. 1, 2 AktG jedenfalls eine Beschlussfassung und damit die Möglichkeit, „andere Aktionäre für die von ihr gewünschte Beschlussfassung zu gewinnen und bei einer Ablehnung ihrer Anträge den entsprechenden Beschluss der Hauptversammlung einer gerichtlichen Nachprüfung zu unterziehen" ${ }^{25}$

Für den Fall, dass der Vorstand einem berechtigten Minderheitsverlangen nach $\$ 122 \mathrm{Abs.} 1,2 \mathrm{AktG}$ nicht nachkommt, kann die Minderheit nach Maßgabe des $\$ 122$ Abs. 3 AktG eine gerichtliche Entscheidung herbeiführen, die die Ermächtigung ausspricht, die Hauptversammlung einzuberufen ( $\$ 122$ Abs. 1 AktG) oder den zusätzlichen Gegenstand bekanntzumachen ( $\$ 122$ Abs. 2 AktG), mithin zur Selbstvornahme zu schreiten. Mit Ausübung dieser gerichtlichen Ermächtigung handelt die Minderheit nicht als Organ der Gesellschaft und schon gar nicht in der Rolle des nach $\$ 121 \mathrm{Abs}$. 2 Satz $1 \mathrm{AktG}$ an sich für die Einberufung zuständigen Vorstands, sondern in Ausübung einer ihr selbst eingeräumten Befugnis und damit aus eigenem Recht und in eigenem Namen. ${ }^{26}$

Ein Aktionär, der gemäß $\ 122$ Abs. 3 AktG gerichtlich zur Einberufung einer Hauptversammlung ermächtigt ist, hat deshalb die Einberufungsformalitäten und Organisationsleistungen in eigener Verantwortung durchführen. Demzufolge hat er die Einberufung im eigenen Namen vorzunehmen ${ }^{27}$ und hierbei die allgemeinen Einberufungsvorschriften mit Ausnahme der Pflicht des Vorstands zur Unterbreitung von Beschlussvorschlägen ( $\mathbb{1 2 4}$ Abs. 3 Satz 1 $\mathrm{AktG})^{28} \mathrm{zu}$ beachten. Die organisatorischen und logistischen Vorbereitungen sind nach der gesetzlichen Konzeption sogar vollständig auf den ermächtigten Aktionär verlagert. ${ }^{29}$ Ausgenommen von dieser Zuständigkeitsüberwälzung sind allein die Einberufungsfolgepflichten der $\mathbb{S} 124 \mathrm{aff}$ AktG aufgrund

24 Statt aller HüFfER, aaO (Fn. 7), \122 AktG Rdn. 1; s. ferner OLG München AG 2010, 84,85 .

25 BGH NJW-RR 2012, 997 Tz. 8; KG NZG 2003, 441, 443; Kubis, aaO (Fn. 11), $\$ 122$ AktG Rdn. 1; Hüffer, aaO (Fn. 7), $\$ 122$ AktG Rdn. 1 .

26 Kubis, aaO (Fn. 11), $\ 122$ AktG Rdn.67, 71; Nonck/Zetzsche, aaO (Fn. 7), \122 AktG Rdn. 108, 115; Rieckers, aaO (Fn. 7), $\$ 122$ AktG Rdn. 67; Werner, aaO (Fn. 7), $\$ 122$ AktG Rdn. 68; aA noch Koch, AG 1969, 1 (Fn. 4); missverständlich WaGner, ZZP 1992, 294, 301 („Notorgan“).

27 Noack/Zetzsche, aaO (Fn. 7), $\$ 122$ AktG Rdn. 115; Kubis, aaO (Fn. 11), $\$ 122$ AktG Rdn. 67; Rieckers, aaO (Fn. 7), $\$ 122$ AktG Rdn. 67; Werner, aaO (Fn. 7), $\$ 123$ AktG Rdn. 68; Butzke, aaO (Fn. 10), Kap. B Rdn. 125.

28 S. unter II. 2. c).

29 S. nur Kubis, aaO (Fn. 11), $\$ 122$ AktG Rdn.71; ebenso Bayer/Scholz/Weiss, AG 2013, 742, 744. 
zwingender entgegenstehender Sachgesichtspunkte. ${ }^{30}$ Diese gesetzliche $\mathrm{Zu}-$ ständigkeitszuweisung rechtfertigt sich insbesondere auch unter dem Aspekt, der - einberufungsunwilligen - Gesellschaft ein möglichst geringes Blockadepotential zu eröffnen. Andererseits kann der gerichtlich ermächtigte Aktionär nach $\$ 122$ Abs. 4 AktG weitestgehend Ersatz der ihm entstandenen Aufwendungen von der Gesellschaft beanspruchen. Im Einzelnen:

\section{b) Einberufung und Vorbereitung, nicht Durchfübrung}

In sachlich-zeitlicher Hinsicht lässt sich die gesetzliche Zuständigkeitszuweisung schlagwortartig mit der Formulierung „Einberufung und Vorbereitung, nicht aber Durchführung “ charakterisieren. Der ermächtigte Aktionär ist zu denjenigen rechtlichen, rechtsgeschäftlichen und tatsächlichen Vorbereitungshandlungen berechtigt und befugt, die bis zur Durchführung einer ordnungsgemäßen Hauptversammlung notwendig werden. Präzisierend bedeutet diese Grenzziehung, dass die Zuständigkeit des ermächtigten Aktionärs dort endet, wo die Zuständigkeit des Versammlungsleiters beginnt. Dessen Zuständigkeit als eines nichtständigen Organs der Gesellschaft ${ }^{31}$ setzt dabei nicht erst mit der Eröffnung der Hauptversammlung ein. Seine Verantwortlichkeit umfasst vielmehr nach ganz h. M. auch schon die Legitimationsprüfung der zur Hauptversammlung erschienenen Aktionäre und die Entscheidung über deren Teilnahme ${ }^{32}$ - auf der Grundlage des als Vorbereitungsmaßnahme erstellten Entwurfs des Teilnehmerverzeichnisses ${ }^{33}$ - und die der Gewährleistung eines ungestörten Hauptversammlungsablaufs dienenden Ordnungsmaßnahmen wie etwa Taschenkontrollen etc. ${ }^{34}$

\section{c) Grundsätzliche Maßgeblichkeit der allgemeinen Einberufungsvorschriften}

Bei der Einberufung hat der ermächtigte Aktionär neben $\$ 122$ Abs. 3 Satz 3 AktG unstreitig die allgemeinen Vorschriften, also insbesondere die $\mathbb{\$} \$ 121$ Abs. 3, 4, 123, 124 Abs. 2 und 3 Satz 4 AktG, zu beachten. ${ }^{35}$ Eine Ausnahme

30 S. unter II. 2. d).

31 Mülbert, Großkomm. z. AktG, 4. Aufl., 1999/2008, Vor $\$ \int 118-146$ Rdn. 86; SchürnBRAND, Organschaft im Recht der privaten Verbände, 2007, S. $142 \mathrm{ff}$.

32 Kubis, aaO (Fn. 11), $\$ 123$ AktG Rdn. 41; RIeckers, aaO (Fn. 7), $\$ 123$ AktG Rdn. 41; Herrler, aaO (Fn. 7), $\$ 123$ AktG Rdn. 7; a. A. - Zuständigkeit des Vorstands - NoAck/Zetzsche, aaO (Fn. 7), \$123 AktG Rdn. 69.

33 Dazu noch unter II. 2. e).

34 Wicke, in: Spindler/Stilz, AktG, 2. Aufl., 2010, Anh $\$ 119$ Rdn. 6; Butzke, aaO (Fn. 10), Kap. D Rdn. 22.

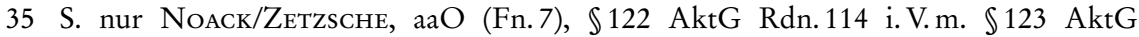
Rdn. 103; Werner, aaO (Fn. 7), $\$ 122 \mathrm{AktG}$ Rdn. 70; HüFfer, aaO (Fn. 7), $\$ 122 \mathrm{AktG}$ 
hiervon bildet nach ganz h. M. die Pflicht zur Unterbreitung von Vorschlägen zur Beschlussfassung in der Einberufung nach $\$ 124$ Abs. 3 Satz 1 AktG. ${ }^{36}$ Soweit es hierzu regelmäßig heißt, dass der kraft gerichtlicher Entscheidung ermächtigte Aktionär hierdurch (ausweislich des $\$ 122 \mathrm{Abs.}_{4} \mathrm{AktG}^{37}$ ) nicht an die Stelle des Vorstands ${ }^{38}$ bzw. eines Organs der Gesellschaft ${ }^{39}$ trete, greift dies freilich zu kurz. Denn bei der Formulierung von Beschlussvorschlägen wird der Vorstand niemals alleine tätig, sondern entweder haben Vorstand und Aufsichtsrat gemeinsam oder, bei der Wahl von Aufsichtsratsmitgliedern und Abschlussprüfern, überhaupt nur der Aufsichtsrat einen Beschlussvorschlag zu machen. Näher liegt daher stattdessen die Erwägung, dass im Falle eines isolierten Ergänzungsverlangens nach $\ 122$ Abs. 2 AktG der von der Minderheit $\mathrm{zu}$ formulierende Beschlussvorschlag keinesfalls die von $\$ 124$ Abs. 3 Satz 1 AktG geforderte Begründungsqualität haben muss ${ }^{40}$ und insoweit den ermächtigten Aktionär keine weitergehenden Pflichten treffen können, wenn er die Beschlussfassung über den Gegenstand im Wege der Einberufung einer Hauptversammlung statt eines bloßen Ergänzungsverlangens durchsetzt. ${ }^{41}$

Die somit ohnehin unter dem Vorbehalt des $₫ 124$ Abs. 3 Satz 1 AktG stehende Maßgeblichkeit der allgemeinen Vorschriften über die Einberufung schließt es nicht aus, dass diese Vorschriften im Falle des $\$ 122$ Abs. 3 AktG nur mit Modifikationen zur Anwendung gelangen. So liegt es auf der Hand und ist schon mit Blick auf die Vorgaben der Aktionärsrechterichtlinie ${ }^{42}$ unabdingbar, dass die Zusatzangaben des $₫ 121$ Abs. 3 Satz 3 AktG im Falle des $\$ 122$ Abs. 3 AktG nicht vom Vorstand oder vom Aufsichtsrat, sondern - abweichend vom Wortlaut - von den einberufenden Aktionären zu machen sind. ${ }^{43}$ Anerkannt

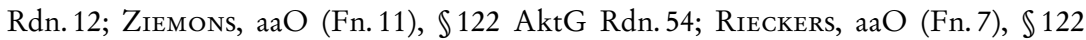
AktG Rdn. 64; Herrler, aaO (Fn. 7), $\$ 122$ AktG Rdn. 18; s. auch Kubis, aaO (Fn. 11), \$122 AktG Rdn. 70: auch $\$ 124$ a AktG.

36 OLG München AG 2010, 84, 87; Kubis, aaO (Fn. 11), \$122 AktG Rdn. 70; Herrler,

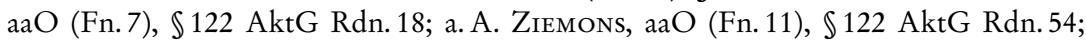
Reichert/Balke, in: Semler/Volhard/Reichert (Hrsg.), Arbeitshandbuch für die Hauptversammlung, 3. Aufl., 2011, 44 Rdn. 59; Butzke, aaO (Fn. 10), Kap. C Rdn. 126.

37 Näher sogleich unter II. 2. f).

38 Werner, aaO (Fn. 7), $\ 122$ AktG Rdn. 70; Rieckers, aaO (Fn. 7), $\$ 122$ AktG Rdn. 64.

39 Noack/Zetzsche, aaO (Fn. 7), $\$ 122$ AktG Rdn. 120.

40 Kubis, aaO (Fn. 11), $₫ 122$ AktG Rdn. 32; s. auch Noack/Zetzsche, aaO (Fn. 7), $\$ 122$ AktG Rdn. 57; a. A. wohl Butzke, aaO (Fn. 10), Kap. B Rdn. 126.

41 A. A. i.E. Reichert/Balke, aaO (Fn. 36), \4 Rdn. 59.

42 Richtlinie 2007/36/EG des Europäischen Parlaments und des Rates über die Ausübung bestimmter Rechte von Aktionären in börsennotierten Gesellschaften vom 11.7.2007, ABl. Nr. L 184/17; für einen Überblick s. GrundmanN, Europäisches Gesellschaftsrecht, 2. Aufl., 2011, \12; HabersaCK/Verse, Europäisches Gesellschaftsrecht, 4. Aufl., 2012, \$7; LutTer/BAYER/J. SCHMidt, Europäisches Unternehmens- und Kapitalmarktrecht, 5. Aufl., 2012, $\$ 31$.

43 Näher Noack/Zetzsche, aaO (Fn. 7), 121 AktG Rdn. 86. 
ist auch, dass in den Fällen des $\$ 122$ Abs. 3 AktG die nach Maßgabe des $\$ 123$ Abs. 2 Satz 3 AktG mögliche Entscheidung über eine Verkürzung der Einberufungsfrist der einberufenden Aktionärsminderheit obliegt. ${ }^{44}$

\section{d) Keine Zuständigkeitsüberwälzung betreffend konkrete Einberufungsfolgepflichten}

Keine Zuständigkeitsüberwälzung ist ferner hinsichtlich der konkret der Ge-

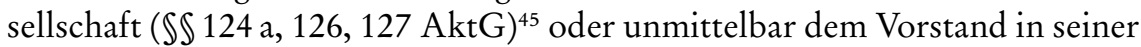
Eigenschaft als für die Gesellschaft handelndem Organ $(\$ 125 \mathrm{AktG})^{46}$ zugewiesenen Einberufungsfolgepflichten anzunehmen. Was die Begründung anbelangt, lässt sich für $\$ 125 \mathrm{AktG}$ wiederum darauf verweisen, dass der ermächtigte Aktionär eben nicht an die Stelle des Vorstands als Organ der Gesellschaft tritt. Letztlich maßgebend erscheint freilich ein anderer, zu $\$ 124$ a AktG vereinzelt im Schrifttum ${ }^{47}$ schon benannter Sachgesichtspunkt, nämlich die zwingende Notwendigkeit einer Mitwirkung der Gesellschaft bei der Erfüllung der Pflichten nach den $\mathbb{S} \$ 124$ aff AktG. Offenkundig ist dies im Falle des $\$ 124$ a AktG, der für börsennotierte Gesellschaften ( $\$ 3$ Abs.2 $\mathrm{AktG}$ ) die Veröffentlichung der Einberufung und weiterer Informationen auf der Internetseite der Gesellschaft vorschreibt. Diese Veröffentlichung auf ihrer eigenen Internetseite kann nur die Gesellschaft selbst leisten, ${ }^{48}$ weil eine Kooperationsverpflichtung der Gesellschaft zur Ermöglichung und Duldung des Zugriffs auf ihre eigene Internetseite ganz und gar unverhältnismäßig und mit Art. 14 Abs. 1 Satz 2 GG nicht zu vereinbaren wäre. Dasselbe gilt für die Veröffentlichung von Anträgen und Wahlvorschlägen von Aktionären, die nach $\$ 126$ Abs. 1 Satz 3 AktG, gegebenenfalls i. V.m. $\$ 127$ Satz 1 AktG, auf der Internetseite der Gesellschaft zugänglich zu machen sind. Aber auch im Falle des $\ 125$ Abs. 1 AktG ist die Mitwirkung der Gesellschaft unverzichtbar, weil bei dieser Mitteilungspflicht sich der Kreis der Mitteilungsadressaten nach den Verhältnissen der letzten Hauptversammlung bemisst.

Muss die Gesellschaft bei der Erfüllung der Pflichten der $\mathbb{S} \$ 124$ aff AktG ohnehin mitwirken und hierbei sogar ganz maßgeblich tätig werden, erscheint der Verzicht auf eine Zuständigkeitsüberwälzung auf den ermächtigten Aktionär sachgerecht und geboten.

44 Drinhausen, aaO (Fn. 7), $\$ 123$ AktG Rdn. 7; Noack/Zetzsche, aaO (Fn. 7), $\$ 123$ AktG Rdn. 110.

45 S. nur Noack/Zetzsche, aaO (Fn. 7), \$122 AktG Rdn. 116; Bayer/Scholz/Weiss, AG 2013, 742, 745; a. A. für $\ 124$ a AktG wohl Kubis, aaO (Fn. 11), $\$ 122 \mathrm{AktG}$ Rdn. 70.

46 S. nur Noack/Zetzsche, aaO (Fn. 7), $\$ 122$ AktG Rdn. 116.

47 Noack/Zetzsche, aaO (Fn. 7), \$122 AktG Rdn. 117.

48 Zutreffend Noack/Zetzsche, aaO (Fn. 7), \$122 AktG Rdn. 117. 


\section{e) Organisation der Hauptversammlung durch den Aktionär im eigenen Namen}

Der ermächtigte Aktionär hat die erforderlichen organisatorischen und logistischen Vorkehrungen für die Durchführung der Hauptversammlung selbst zu treffen; ${ }^{49}$ die Gesellschaft ist über die Erfüllung der ihr gesetzlich zugewiesenen Einberufungsfolgepflichten bei wirksamer Einberufung ( $\mathbb{S} 124 \mathrm{aff}$ AktG) hinaus zu keinen Mitwirkungshandlungen verpflichtet und kann ganz untätig bleiben..$^{50}$

Diese Pflichtzuständigkeit des ermächtigten Aktionärs für die organisatorische Vorbereitung der Hauptversammlung beinhaltet auch die Vorbereitung des Teilnehmerverzeichnisses ( $\$ 129$ Abs. 1 Satz 2, Abs. 4 AktG). Das wird im Schrifttum zwar nicht ausdrücklich ausgesprochen - auch weil dies als schiere Selbstverständlichkeit erscheinen mag -, ergibt sich aber ohne weiteres aus folgenden Überlegungen: Das Teilnehmerverzeichnis ist ausweislich des $\$ 129$ Abs. 1 Satz 2 AktG ,in der Hauptversammlung “ aufzustellen. Ein Entwurf ist jedoch bereits im Vorfeld anzufertigen, schon um ihn der Einlasskontrolle zugrunde legen zu können und ihn unter Abgleich mit den tatsächlich erschienen Aktionären in das Teilnehmerverzeichnis zu überführen. ${ }^{51}$ Hierbei handelt es sich um eine Vorbereitungsmaßnahme infolge der Anmeldepflicht des $\$ 123$ Abs. 2 AktG. ${ }^{52}$ Mit der vollumfänglichen Überwälzung der organisatorischen und logistischen Vorbereitungen der Hauptversammlung auf den ermächtigten Aktionär obliegt diesem auch die Erstellung eines Entwurfs des Teilnehmerverzeichnisses, wozu er mittels Mitteilung einer entsprechenden Zugangsadresse - gegebenenfalls eines Hauptversammlungs-Dienstleisters als empfangsberechtigter Anmeldestelle - auch in der Lage ist.

Soweit die organisatorische Vorbereitung den Abschluss entsprechender Rechtsgeschäfte erfordert - etwa für die Beauftragung eines Notars, die Anmietung eines Versammlungslokals, die Bereitstellung technischer Hilfsmittel, die Beauftragung von Ordnungskräften und die Bewirtung -, hat der Aktionär diese im eigenen Namen zu tätigen. ${ }^{53}$ Die gerichtliche Ermächtigung hat nicht etwa zur Folge, dass der Aktionär im Hinblick auf Vorbereitung und Durch-

49 Werner, aaO (Fn. 7), $\ 122$ AktG Rdn. 73; Rieckers, aaO (Fn. 7), $\$ 122$ AktG Rdn. 67; Herrler, aaO (Fn. 7), $\$ 122$ AktG Rdn. 18; Reichert/Balke, aaO (Fn. 36), $\$ 4$ Rdn. 61.

50 Allg. M.; s. etwa Kubis, aaO (Fn. 11), $\$ 122$ AktG Rdn. 71; Rieckers, aaO (Fn. 7), $\$ 122$ AktG Rdn. 67; auch Noack/Zetzsche, aaO (Fn. 7), \$122 AktG Rdn. 116.

51 S. nur Kubis, aaO (Fn. 11), $\$ 129$ AktG Rdn. 16; 18; Hüffer, aaO (Fn. 7), $\$ 129$ AktG Rdn. 8; Werner, aaO (Fn. 7), \$129 AktG Rdn. 13; Noack/Zetzsche, aaO (Fn. 7), \$129 AktG Rdn. 74; Wicke, aaO (Fn. 34), $\$ 129$ AktG Rdn. 23.

52 So wörtlich Kubis, aaO (Fn. 11), $\ 129$ AktG Rdn. 16.

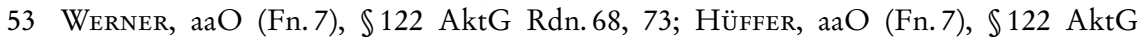
Rdn. 12; Zöllner, Kölner Komm. z. AktG, 1. Aufl., 1985, $\$ 122$ Rdn. Rdn. 49; RieCKers, aaO (Fn. 7), $\$ 122$ AktG Rdn.67; Reger, in: Bürgers/Körber, AktG, 2. Aufl., 
führung der Hauptversammlung an die Stelle des Vorstands der Gesellschaft träte ${ }^{54}$ und diese also organschaftlich vertreten könnte. ${ }^{55}$ Das folgt ohne weiteres daraus, dass $\mathbb{1 2 2}$ Abs. 4 AktG eine Regelung zur Erstattung der dem Aktionär entstandenen Aufwendungen trifft. Denn eine solche wäre offenkundig entbehrlich, könnte der Aktionär die Gesellschaft rechtsgeschäftlich wirksam vertreten.

\section{f) Erstattungsanspruch gemäß $\ 122 \mathrm{Abs.} 4 \mathrm{Akt} G$}

Nach der vorstehend angesprochenen Vorschrift des $\ 122$ Abs. 4 AktG hat die Gesellschaft einem Aktionär die Aufwendungen zu ersetzen, die diesem aus der Durchführung einer von ihm kraft gerichtlicher Ermächtigung einberufenen Hauptversammlung entstehen. Die Regelung impliziert damit das Bestehen eines entsprechenden Erstattungsaufwands bzw. gewährt selbst einen solchen. ${ }^{56}$ Verbreitet wird dem ermächtigenden Aktionär darüber hinaus, wenn auch zumeist ohne Nennung einer Rechtsgrundlage - in Betracht kommt etwa $\$ 257 \mathrm{BGB}$-, ein Freistellungsanspruch gegen die Gesellschaft zuerkannt. ${ }^{57}$

Was die Anspruchshöhe anbelangt, wird vereinzelt eine Beschränkung unter Orientierung an den von der Gesellschaft selbst praktizierten Gewohnheiten ${ }^{58}$ oder auf den üblichen Rahmen ${ }^{59}$ befürwortet. Vorzugswürdig dürfte dem-

2011, $\ 122$ Rdn. 23; a.A. für die Beauftragung des Notars - durch den Vorstand Noack/Zetzsche, aaO (Fn. 7), $\$ 122$ AktG Rdn. 116.

54 Ganz h. M.; s. nur Zöllner, aaO (Fn. 53), $\$ 122$ AktG Rdn. 43, 49; Werner, aaO (Fn.7), \$122 AktG Rdn.68; Noack/Zetzsche, aaO (Fn.7), \$122 AktG Rdn.120; Kubis, aaO (Fn.11), $\$ 122$ AktG Rdn.67, 71; Rieckers, aaO (Fn.7), $\mathbb{1} 122$ AktG Rdn. 62; Butzke, aaO (Fn. 10), Kap. B Rdn. 125; a. A. vor allem Koch, AG 1969, 1 in Fn. 4.

55 Ganz h. M., s. HüFfER, aaO (Fn. 7), \122 AktG Rdn. 12; Zöllner, aaO (Fn. 53), \122 AktG Rdn.49; Noack/Zetzsche, aaO (Fn.7), $\$ 122$ AktG Rdn.128; Kubis, aaO (Fn. 11), $\$ 122$ AktG Rdn.71; Mayrhofer, in: Wachter, AktG, 2012, $\mathbb{1 2 2}$ Rdn. 25; mit anderer Begründung - keine Ermächtigung durch $\$ 122 \mathrm{Abs} .4 \mathrm{AktG}$ - auch REgER, aaO (Fn. 53), \$122 AktG Rdn. 23 (zweifelhaft).

56 Im zweiten Sinne etwa Noack/Zetzsche, aaO (Fn. 7), $\$ 122$ AktG Rdn. 126; Hüffer, aaO (Fn. 7), \122 AktG Rdn. 13; Kubis, aaO (Fn. 11), \122 AktG Rdn. 73; Ziemons, aaO (Fn. 11), \122 AktG Rdn. 55; ohne Stellungnahme etwa Werner, aaO (Fn. 7), \$122 AktG Rdn. 77 ff; Rieckers, aaO (Fn.7), $\$ 122$ AktG Rdn.69; Herrler, aaO (Fn.7), $\$ 122$ AktG Rdn. 19

57 NoAck/Zetzsche, aaO (Fn. 7), \122 AktG Rdn. 126 (aus $\$ 122$ Abs. 4 AktG); Hüffer,

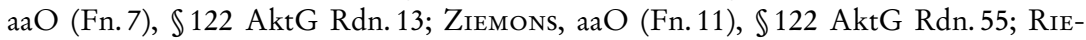
CKers, aaO (Fn. 7), \$122 AktG Rdn. 69; Herrler, aaO (Fn. 7), \122 AktG Rdn. 19; Halberkamp/Gierke, NZG 2004, 494, 501.

58 Kubis, aaO (Fn. 11), $\$ 122$ AktG Rdn. 73.

59 Rieckers, aaO (Fn. 7), $\$ 122 \mathrm{AktG}$ Rdn. 69. 
gegenüber eine Begrenzung dergestalt sein, dass es dem Aktionär in Extremfällen, etwa der Auswahl eines im Marktvergleich offenkundig überteuerten Anbieters, unter Rückgriff auf seine Treuepflicht gegenüber der Gesellschaft versagt wird, seinen Erstattungs- bzw., bei dessen Anerkennung, seinen Freistellungsanspruch in voller Höhe geltend zu machen.

Keine Zustimmung verdienen vor diesem Hintergrund die wenigen Gegenstimmen, die $₫ 122$ Abs. 4 AktG als gesetzliche Anordnung einer Verpflichtungsermächtigung deuten wollen. ${ }^{60}$ Der hierfür in Stellung gebrachte Hinweis, dass nur hierdurch der Streit über Art und Höhe der Freistellung und Zahlung beseitigt und der vom Gesetzgeber gewollte effiziente Minderheitenschutz gewährleistet werde, ${ }^{61}$ blendet zu Unrecht aus, dass die Verpflichtungsermächtigung im allgemeinen Zivilrecht als Durchbrechung des für die Vollmacht geltenden Offenkundigkeitsgrundsatzes ( $\$ 164$ Abs. 1 BGB) aus Gründen des Verkehrsschutzes für unzulässig angesehen wird ${ }^{62}$ und dass diese Erwägungen zum Schutze des Dritten davor, nach Vertragsschluss eine andere Partei als seinen Vertragspartner präsentiert zu bekommen, auch für die hier in Frage stehenden Rechtsgeschäfte Geltung beanspruchen können. Im vorliegenden Kontext gilt dies sogar in besonderem Maße, weil der Dritte gerade nicht unter etwaigen Auseinandersetzungen zwischen der Gesellschaft und dem ermächtigten Aktionär über die Berechtigung eines Rechtsgeschäfts leiden darf.

\section{Befugnis des ermächtigten Aktionärs zur Benennung einer Anmeldestelle im Besonderen}

\section{Diskussionsstand}

Was die im Folgenden zu klärende Frage anbelangt, ob die Ermächtigung der Aktionärsminderheit zur Einberufung der Hauptversammlung auch das Recht umfasst, eine Anmeldestelle zu benennen, so enthält sich das AktG einer eindeutigen Regelung. Rechtsprechung zu dieser Frage liegt, soweit ersichtlich, bislang nicht vor, und auch das Schrifttum behandelt die Frage stiefmütterlich. Soweit sich in der Kommentarliteratur hierzu überhaupt Aussagen finden, heißt es dazu: „Die Auswahl der Adresse trifft der Einberufende, i. d. R. also der Vorstand ( $\mathbb{1 2 1}$ Abs. 2 Satz 1$)$, in den Fällen des $₫ 122$ Abs. 3 Satz 1 Hs. 1 die Aktionärsminderheit" ${ }^{\text {" }}{ }^{63}$ Wie die Gegenüberstellung von Vorstand und Aktionärsminderheit unzweideutig erkennen lässt, ist der zur Ein-

60 Insbesondere Pluta, aaO (Fn. 7), $\$ 123$ AktG Rdn. 36.

61 Pluta, aaO (Fn. 7), \123 AktG Rdn. 36

62 S. nur BGHZ 114, 96, 100 = NJW 1985, 1815; Ellenberger, aaO (Fn. 13), $\$ 185$ BGB Rdn. 3; Bayreuther, Münchener Komm. z. BGB, 6. Aufl., 2012, \185 Rdn. 31.

63 Noack/Zetzsche, aaO (Fn. 7), \$123 AktG Rdn. 103. 
berufung ermächtigte Aktionär nach dieser Ansicht frei in der Wahl einer Anmeldeadresse und bedarf auch bei Benennung einer Anmeldestelle nicht der Zustimmung der Gesellschaft. Nachdem auch Noack/Zetzsche - mit der wohl einhelligen Ansicht - ausdrücklich betonen, dass die Zugangsadresse keine Adresse der Gesellschaft sein muss, vielmehr auch ein Dienstleister benannt werden kann, ${ }^{64}$ kann das nur bedeuten, dass nach Ansicht der Autoren die Aktionärsminderheit die Adresse eines von ihr bestimmten Dienstleisters anzugeben berechtigt ist.

Eine abweichende Position haben jüngst Bayer/Scholz/Weiß eingenommen. ${ }^{65}$ Ihrer Ansicht nach widerspricht die Angabe einer nicht mit der Gesellschaft abgestimmten Anmeldeadresse bereits dem Wortlaut des $\$ 123$ Abs. 2 Satz 2, Abs. 3 Satz 3 AktG, denen zufolge Anmeldung und Nachweis „der Gesellschaft" zugehen müssten. Aber auch der Zweck des Anmeldeerfordernisse spreche gegen die Befugnis der ermächtigten Aktionärsminderheit zur Bestimmung einer Anmeldeadresse, sei doch nur so gewährleistet, dass der Versammlungsleiter der ihm obliegenden Legitimationsprüfung nachkommen könne. Der Gefahr, dass der - zur Einberufung nicht willige - Vorstand der Gesellschaft oder der Versammlungsleiter die ordnungsgemäße Einberufung der Hauptversammlung torpediere, könne ohne Weiteres dadurch begegnet werden, dass das Gericht nach $\$ 122$ Abs. 3 Satz 2 AktG nicht nur zur Einberufung ermächtigt, sondern darüber hinaus den Versammlungsleiter bestimmt.

\section{Geringe Aussagekraft des Wortlauts des $\int 123$ Abs. 2 Satz 2 Akt $G$}

Für die Stellungnahme erweist sich der Wortlaut des $\$ 123$ Abs. 2 Satz 2 AktG als wenig aussagekräftig. Zwar verlangt die Vorschrift, dass die Anmeldung „der Gesellschaft unter der in der Einberufung hierfür mitgeteilten Adresse mindestens sechs Tage vor der Versammlung zugehen" muss. Doch ist es gerade die Frage, ob das Erfordernis eines Zugangs bei der Gesellschaft auch für die - in der Praxis und auch regelungssystematisch den Ausnahmefall bildende - Einberufung durch den gerichtlich ermächtigten Aktionär gilt. Die Tatsache jedenfalls, dass die Einladung zur Hauptversammlung durch den - im eigenen Namen und in Ausübung eines eigenen Rechts handelnden - ermächtigten Aktionär zu erfolgen hat und diesem, wie bereits dargelegt worden ist ${ }^{66}$ auch die Vorbereitung der Hauptversammlung obliegt, nährt jedenfalls Zweifel daran, dass der Gesetzgeber in $\$ 123$ Abs. 2 Satz 2 AktG auch den Fall der Einberufung gemäß $\$ 122$ Abs. 3 AktG regeln wollte. Be-

64 Noack/Zetzsche, aaO (Fn. 7), \$123 AktG Rdn. 107.

65 Bayer/Scholz/WeIss, AG 2013, 742, 746 ff (gleichfalls auf ein Rechtsgutachten Bayers zurückgehend).

66 S. im Einzelnen unter II. 2. 
denkt man weiter, dass das Gesetz ein Anmeldeerfordernis überhaupt nicht kennt, „Ob“ und „Wie“ eines solchen Erfordernisses vielmehr - vorbehaltlich der Vorgaben in $\$ 123$ Abs. 2 Satz 2 bis 4 AktG - in die Hände des Satzungsgebers gelegt werden, zeigt sich rasch, dass ein Hinweis auf den Wortlaut des $₫ 123$ Abs. 2 Satz 2 AktG die Problematik nicht vollständig erfasst, die Frage vielmehr in den größeren Zusammenhang der Vorbereitung und Durchführung der nach Maßgabe des $\$ 122$ Abs. 3 AktG einberufenen Hauptversammlung zu stellen ist und nicht ohne Berücksichtigung systematischer und teleologischer Erwägungen beantwortet werden kann, zumal aufgezeigt werden konnte, dass auch andere Vorschriften über die Einberufung im Falle des $₫ 122$ Abs. 3 AktG nur mit Modifikationen zur Anwendung zu bringen sind. ${ }^{67}$

\section{Die Aktionärsbefugnis als Ausfluss der gesetzlichen Grundkonzeption}

Der gerichtlich zur Einberufung ermächtigte Aktionär ist, dies konnte bereits festgestellt werden, nach der gesetzlichen Konzeption für die Vorbereitungen der Hauptversammlung in organisatorischer und logistischer Hinsicht vollumfänglich und in rechtlicher Hinsicht vorbehaltlich des $\$ 124$ Abs. 3 Satz 1 AktG sowie der Einberufungsfolgepflichten ( $\$ \$ 124$ aff AktG) zuständig ${ }^{68}$ und hat daher auch die $\mathbb{S} 121$ Abs. 3, 4, 123, 124 Abs. 2 und 3 Satz 4 AktG zu beachten. ${ }^{69}$ Aus dieser gesetzlichen Grundkonzeption folgt bei unbefangenem Zusehen ohne weiteres, dass er in der Einberufung für den Zugang eine Adresse benennen und hierbei gemäß den allgemeinen Grundsätzen ${ }^{70}$ auch eine Anmeldestelle unter einer c/o-Adresse einschalten darf, ohne auf das Einverständnis der Gesellschaft hiermit angewiesen zu sein. Es müsste merkwürdig anmuten, könnte die Gesellschaft dem gerichtlich ermächtigten Aktionär vorgeben, welchen Hauptversammlungsdienstleister er (nicht) verpflichten darf, obwohl das Anmeldeerfordernis (sofern es überhaupt besteht) nur die Vorbereitung der Hauptversammlung erleichtern soll und die ermächtigte Aktionärsminderheit bei Wahrnehmung ihrer Ermächtigung - und damit bei der Vorbereitung der Hauptversammlung - in eigenem Namen handelt.

Soweit die Gesellschaft das Anmeldeerfordernis qua Satzung eingeführt hat, sie aber abweichend vom Regelfall für die Vorbereitung der Hauptversammlung gar nicht verantwortlich ist, wäre die Anmeldung gegenüber der Gesellschaft - vorbehaltlich der der Gesellschaft auch in den Fällen des $\$ 122 \mathrm{Abs.} 3$

67 S. unter II. 2. c); verkürzt deshalb die auf den Wortlaut des $₫ 122$ Abs. 2 Satz 2 AktG bezogene Argumentation von Bayer/Scholz/WeIss, AG 2013, 742, $746 \mathrm{f}$.

68 S. unter II. 2.

69 S. unter II. 2. c).

70 S. unter II. 1. b). 
obliegenden Legitimationsprüfung ${ }^{71}$ - sinn- und funktionslos. In dieser Konstellation wird der Zweck des Anmeldeerfordernisses vielmehr allein gewahrt, wenn die Anmeldung gegenüber dem für die Vorbereitung der Hauptversammlung Verantwortlichen erfolgt, in den Fällen des $₫ 122$ Abs. 3 AktG mithin gegenüber der ermächtigten Aktionärsminderheit.

Daraus wiederum folgt nicht nur, dass die Minderheit bei Ausübung der Ermächtigung zur Einberufung eine ihr genehme Anmeldeadresse bestimmen kann, sondern gleichermaßen, dass es sich bei der in der Einberufung genannten Anmeldestelle um einen bevollmächtigten Empfangsvertreter der Gesellschaft oder jedenfalls einen mit Botenmacht versehener Empfangsboten der Gesellschaft handeln muss. Konsequent zu Ende gedacht ergibt sich hieraus, dass die Kompetenz der einberufenden Aktionärsminderheit zur Angabe einer Anmeldestelle gesetzliche Folge des ermächtigenden Gerichtsbeschlusses ist, was wiederum bedeutet, dass es sich um einen Fall gesetzlicher Vertretungsbefugnis handelt, die den Aktionär in die Lage versetzt, die Anmeldestelle im Namen der Gesellschaft mit unmittelbarer Wirkung für und gegen diese $\mathrm{zu}$ benennen.

\section{Mögliche Bedenken}

Vorstehendes Ergebnis ist zunächst gegen einige denkbare Einwände abzusichern.

\section{a) Keine Befugnis zur Erteilung einer Empfangsbefugnis mit Wirkung für die Gesellschaft?}

Gegenüber den bisherigen Überlegungen könnte man zunächst einwenden wollen, dass die Einschaltung eines Empfangsvertreters oder Empfangsboten nach den für den Zugang gemäß $\$ 130$ BGB geltenden allgemeinen Regeln ${ }^{72}$ erfordert, dass der Empfänger eine entsprechende Empfangsvollmacht oder Empfangsbotenmacht erteilt hat und dass dies nur dem Vorstand der Gesellschaft (arg. $\$ 78 \mathrm{AktG}$ ), nicht aber dem gerichtlich ermächtigten Aktionär möglich ist. Denn dieser tritt unstreitig gerade nicht an die Stelle des Vorstands und erlangt durch die gerichtliche Ermächtigung auch keine rechtsgeschäftliche Vollmacht zum Abschluss von Rechtsgeschäften für die Gesellschaft. ${ }^{73}$ Die Empfangsbefugnis der Anmeldestelle müsste danach vielmehr notwendig vom Vorstand namens der Gesellschaft erteilt werden.

71 Dazu im vorliegenden Kontext noch unter 4. b).

$72 \mathrm{Zu}$ diesen nur Ellenberger, aaO (Fn. 13), \$130 BGB Rdn. $8 \mathrm{f}$.

73 S. unter II. 2. e). 
Bei näherem Zusehen greift diese Argumentation aber zu kurz. Dass dem ermächtigten Aktionär keine Vertretungsmacht zum Abschluss von Rechtsgeschäften im Namen der Gesellschaft zukommt, ist lediglich dem Umstand geschuldet, dass $\$ 122$ Abs. 4 AktG einen Erstattungsanspruch des bevollmächtigen Aktionärs voraussetzt, was die Annahme einer Bevollmächtigung des ermächtigten Aktionärs zum Abschluss von Rechtsgeschäften im Namen der Gesellschaft ausschließt. ${ }^{74}$ Weitergehende Aussagen dazu, ob der ermächtigte Aktionär für die Gesellschaft anderweitig handeln kann, sind der Vorschrift des $\$ 122 \mathrm{Abs.} 4 \mathrm{AktG}$ nicht zu entnehmen. Im Schrifttum wird sogar, wenn auch unter teleologischen Aspekten wenig überzeugend, aus $\mathbb{} 122$ Abs. 4 AktG entnommen, dass dem ermächtigten Aktionär eine Verpflichtungsermächtigung zukomme. ${ }^{75}$ Erst recht lässt sich aus $\$ 122 \mathrm{Abs.} 4 \mathrm{AktG}$ nicht ableiten, dass der zur Einberufung ermächtigte Aktionär nicht schon entsprechend des hiesigen Verständnisses ${ }^{76}$ kraft Gesetzes bzw., noch genauer, kraft der gerichtlichen Ermächtigung zur Einberufung einer Hauptversammlung nach Maßgabe der $\mathbb{S} 121 \mathrm{ff}$ AktG eine Anmeldestelle als Empfangsbevollmächtigte bzw. Empfangsbotin unabhängig von der Zustimmung der Gesellschaft benennen kann.

\section{b) Möglichkeit der Selbstbenennung und sonstige Manipulationspotentiale als Einwand?}

Nach der gesetzlichen Grundkonzeption, wie sie nicht zuletzt in $\$ 122$ Abs. 2 i. V. m. den $\int \ 121$ Abs. 3, 4, 123, 124 Abs. 2 und 3 Satz 4 AktG zum Ausdruck kommt, kann der ermächtigte Aktionär auch sich selbst als Anmeldestelle benennen. Auch abgesehen davon, dass von dieser Möglichkeit jedenfalls bei börsennotierten Gesellschaften schon aus praktischen Erwägungen heraus kein Gebrauch gemacht werden wird, ${ }^{77}$ verbinden sich mit ihr in rechtlicher Hinsicht keine Bedenken gegen die alleinige Befugnis des ermächtigten Aktionärs zur Benennung der Anmeldestelle.

Zunächst einmal gilt es nämlich festzuhalten, dass der ermächtigte Aktionär typischerweise ein ungleich größeres Interesse an der fehlerfreien Durchführung der Hauptversammlung und einer mängelfreien Beschlussfassung hat als die - gerichtsnotorisch einberufungsunwillige - Gesellschaft. Sodann ist nicht zu erkennen, dass dem Aktionär aufgrund einer von ihm vorgenommenen Benennung eines Hauptversammlungs-Dienstleisters als Anmeldestelle ein Manipulationsspielraum erwüchse, den es mit der Zuweisung dieser Befugnis an die Gesellschaft zu unterbinden gälte. Zwar ist nicht völlig ausgeschlossen,

74 S. unter II. 2. f).

75 Hierzu ablehnend oben unter II. 2. f).

76 S. unter III. 3.

77 Dazu noch unter III. 5. 
dass der einberufende Aktionär je nach Entwicklung der Anmeldungen zur Hauptversammlung zur Überzeugung gelangt, dass er für seine Anliegen bei der Beschlussfassung nicht die erforderlichen Mehrheiten finden wird und daher die Hauptversammlung noch im Vorfeld wieder absagt. Kostengesichtspunkte sprechen freilich dagegen, weil dem ermächtigten Aktionär ein Erstattungsanspruch gemäß $\$ 122 \mathrm{Abs} .4 \mathrm{AktG}$ zu versagen ist, wenn es trotz Wahrnehmung der Ermächtigung nicht zur Durchführung der Hauptversammlung gekommen ist. ${ }^{78}$

Vor diesem Hintergrund geht auch der denkbare Einwand fehl, der Aktionär könnte bei Annahme einer exklusiven Kompetenz zur Benennung und Beauftragung einer Anmeldestelle die vom Versammlungsleiter vorzunehmende Legitimationsprüfung ${ }^{79}$ erschweren oder gar vereiteln, indem er diesem den alsbald erstellten Entwurf des Teilnehmerverzeichnis nicht rechtzeitig oder nicht ordnungsgemäß zuleitet. De iure ist der Versammlungsleiter ohnehin nicht darauf angewiesen, dass er innerhalb der Anmeldefrist Kenntnis hinsichtlich des Anmeldestandes erlangt. Denn tatsächlich hat die Legitimationsprüfung in den Fällen des $\$ 122$ Abs. 3 AktG jedenfalls auch durch den ermächtigten Aktionär zu erfolgen, der im Zusammenhang mit der Auswahl geeigneter Räume, vor allem aber im Zusammenhang mit der Versendung der Eintrittskarten wissen muss, welche Aktionäre hinreichend legitimiert sind und deshalb zuzulassen sind. Lassen sich somit Anmeldung und Legitimationsprüfung nicht voneinander trennen, kann dies nur bedeuten, dass die dem Versammlungsleiter obliegende Legitimationsprüfung nachgelagert erfolgt, nämlich nach Entgegennahme der Anmeldungen und nach Versand der Eintrittskarten an die hinreichend legitimierten Aktionäre. Bei Lichte betrachtet ist es denn auch im Regelfall des $\$ 121 \mathrm{Abs.} 2$ Satz $1 \mathrm{AktG}$, in dem Einberufung und Vorbereitung der Hauptversammlung dem Vorstand obliegen, keineswegs gewährleistet - und weder gesetzlich noch satzungsmäßig geboten -, dass der Versammlungsleiter (dem die Legitimationsprüfung obliegt) noch vor Anmeldefrist Kenntnis hinsichtlich des Anmeldestandes erlangt. Auch im Regelfall des $\ 121$ Abs. 2 Satz 1 AktG bedarf es vielmehr der Weiterleitung des Anmeldestandes durch den Vorstand (bzw. durch den vom Vorstand eingesetzten Dienstleister), die praktischerweise erst nach Ablauf der Anmeldefrist erfolgt. Im Ausnahmefall des $\ 122$ Abs. 3 AktG besteht allein die Besonderheit, dass nicht der Vorstand, sondern der ermächtigte Aktionär (bzw. der von ihm eingesetzte Dienstleister) den Anmeldestand entgegennimmt und ihn an den Versammlungsleiter weiterleitet.

78 Nicht im Widerspruch hierzu steht daher die Zubilligung eines Kostenersatzanspruchs für den Fall, dass der Vorstand die Einberufung alsbald nach der stattgebenden gerichtlichen Entscheidung übernimmt, wie dies etwa NoAck/Zetzsche, aaO (Fn. 7), $\$ 122$ AktG Rdn. 129 befürworten.

79 S. unter II. 2. b). 
Berücksichtigt man, dass in den Fällen des $\ 122$ Abs. 3 AktG der Vorstand das Einberufungsverlangen der Minderheit notwendigerweise zu Unrecht abgelehnt hat, lässt sich kaum in Abrede stellen, dass die Vorbereitung der von der Aktionärsminderheit mit gerichtlicher Hilfe einberufenen Hauptversammlung - und damit auch die Angabe einer Anmeldeadresse - nicht zuletzt deshalb den ermächtigten Aktionären obliegen muss, weil derjenige, der zwar an sich zuständig ist ( $\mathbb{S} 121 \mathrm{Abs} .2 \mathrm{Satz} 1,122 \mathrm{Abs} .1 \mathrm{Satz} 1 \mathrm{AktG})$, indes gegen seine Überzeugung und seinen Willen handeln müsste, im Allgemeinen kein guter Geschäftsführer ist. Dies gilt zumal in Fällen, in denen die Hauptversammlung über die Wahl neuer Aufsichtsratsmitglieder Beschluss fassen soll und nicht auszuschließen ist, dass der solchermaßen neu gewählte Aufsichtsrat von seiner Personalkompetenz in Bezug auf den Vorstand Gebrauch macht. Ein massiverer Interessenkonflikt in der Person des mit der Vorbereitung der Hauptversammlung befassten Vorstands erscheint kaum vorstellbar. Gut vorstellbar ist hingegen, dass der mit der Vorbereitung der Hauptversammlung befasste Vorstand nach Möglichkeiten sucht, die Durchführung der Hauptversammlung zu torpedieren. Berücksichtigt man weiter, dass eine von der Gesellschaft nach Maßgabe des $\$ 122$ Abs. 3 Satz 4 AktG eingelegte Beschwerde keine aufschiebende Wirkung hat, ${ }^{80}$ erscheint die Vorstellung, der Vorstand komme seiner Pflicht zur Einberufung und Vorbereitung einer Hauptversammlung, die abzuwenden er vermittels der von ihm eingelegten Beschwerde begehren könnte, mit der gebotenen Sorgfalt nach, als nicht eben naheliegend. Denn es ist gerade die ermächtigte Aktionärsminderheit - dies ist nochmals zu betonen -, der an einer gesetzesmäßigen Durchführung der Hauptversammlung und einer bestandskräftigen Beschlussfassung interessiert ist. Ihr ist entgegen der Ansicht von Bayer/Scholz/Wei $\beta^{81}$ auch nicht damit geholfen, dass sie nach $\$ 122 \mathrm{Abs.} 3$ Satz 2 AktG auf die gerichtliche Bestimmung des Versammlungsleiters hinwirkt. In Frage steht nämlich weder die Leitung der Hauptversammlung noch die Legitimationsprüfung, sondern einzig und allein die Vorbereitung der Hauptversammlung nebst Ingangsetzung des Anmeldeverfahrens.

\section{Die Bestätigung}

Für eine auch teleologisch fundierte Bestätigung des hiesigen Gesetzesverständnisses, dass der Aktionär mit der gerichtlichen Ermächtigung zur Einberufung der Hauptversammlung kraft Gesetzes zugleich berechtigt ist, die empfangsberechtigte Anmeldestelle i.S.d. $\$ 123$ Abs. 2 Satz 1 AktG mit Wirkung für die Gesellschaft zu benennen, sind als Ausgangspunkt die Konsequenzen in den Blick zu nehmen, die die - angenommene - Notwendigkeit

80 Vgl. $\$ 64$ Abs. 3 FamFG; ferner statt aller Kubis, aaO (Fn. 11), 122 AktG Rdn. 64.

81 Bayer/Schmidt/Weiss, AG 2013, 742, $747 \mathrm{f}$. 
eines Einverständnisses der Gesellschaft mit der Auswahlentscheidung des ermächtigten Aktionärs hätte.

Ein rechtliches Hindernis für die Einberufung der Hauptversammlung bestünde allerdings nicht. Hat die Gesellschaft ihre Zustimmung zur Benennung einer empfangsberechtigten Anmeldestelle nicht erteilt oder gar endgültig verweigert, kann der Aktionär eine mangelfreie Einberufung dadurch vornehmen, dass er auf die Mitteilung einer Anmeldeadresse ganz verzichtet, weil für diesen Fall ein wirksamer Zugang der Anmeldung an der Postanschrift der Gesellschaft bzw. des Ortes ihrer Hauptverwaltung erfolgen kann.

Mag auch die Gesellschaft die Einberufung der Hauptversammlung nicht rechtlich blockieren können, käme ihr mit der Nichterteilung bzw. der Verweigerung der Zustimmung zur Einschaltung des vom ermächtigten Aktionär präferierten Hauptversammlungs-Dienstleisters gleichwohl ein insbesondere bei börsennotierten Gesellschaften zum Tragen kommendes Blockadepotential zu. Den entscheidenden Grund hierfür hat Butzke auf den Punkt gebracht: Aktionäre, die aufgrund einer gerichtlichen Ermächtigung nach $₫ 122$ Abs. 3 AktG „die Initiative ergreifen und nicht auf die Unterstützung der Gesellschaft setzen können, werden [...] kaum ohne professionelle Hilfe bei der Organisation auskommen". ${ }^{82}$ Bei dieser Sachlage spricht schon das in $\$ 122$ AktG zum Ausdruck kommende gesetzgeberische Regelungsanliegen, das der einberufungsunwilligen Gesellschaft zu Gebote stehende Obstruktionspotential möglichst zu minimieren, ${ }^{83}$ für die alleinige Zuständigkeit des ermächtigten Aktionärs zur Benennung einer Anmeldestelle. Hinzu kommt, nicht minder gewichtig, die ratio des $\$ 123 \mathrm{Abs}$. 2 Satz $2 \mathrm{AktG}$, die organisatorische und logistische Durchführung der Hauptversammlung möglichst zu erleichtern bzw., im Falle vor allem börsennotierter (Publikums-)Gesellschaften, deren Durchführung überhaupt erst zu ermöglichen. ${ }^{84}$ Diese ratio beansprucht gleichermaßen Geltung für den ermächtigten Aktionär, auf den nach der gesetzlichen Konzeption die organisatorische und logistische Vorbereitung der Hauptversammlung vollumfänglich verlagert ist ${ }^{85}$ und der folglich über die gleichen rechtlichen Gestaltungsmittel zur Bewältigung dieser Aufgabe wie die Gesellschaft selbst verfügen muss.

Demgegenüber einwenden zu wollen, dass der ermächtigte Aktionär im Wege einstweiligen Rechtsschutzes gegen die Gesellschaft vorgehen könnte, um zunächst deren Einverständnis mit der Benennung eines Hauptversammlungs-Dienstleisters durchzusetzen und sodann gegebenenfalls die Erteilung der für die ordnungsmäßige Vorbereitung der Hauptversammlung erforderlichen Informationen durch die Gesellschaft zu erstreiten, hieße die Zusam-

82 Butzke, aaO (Fn. 10), Kap. B Rdn. 28.

83 S. unter III. 4.

84 S. Text bei Fn. 82.

85 S. unter II. 2. a), b). 
menhänge auf den Kopf zu stellen und wäre schon im Ansatz verfehlt. Denn nach der gesetzlichen Grundkonzeption ist der ermächtigte Aktionär der für die Einberufung und Vorbereitung der Hauptversammlung verantwortliche Akteur, wogegen die Gesellschaft - sieht man von den Einberufungsfolgepflichten der $\$ \mathbb{S 1 2 4}$ aff AktG ab - untätig bleiben darf. ${ }^{86}$ Dem ermächtigten Aktionär als Ausnahme hiervon die Befugnis zur Benennung einer Anmeldestelle zu versagen und diese der Gesellschaft zuzuweisen, bedürfte gewichtiger Sachgründe, die freilich nicht ersichtlich sind, zumal das Gesetz die mit der Einberufung und Vorbereitung der Hauptversammlung verbundenen Lasten in $\$ 122 \mathrm{Abs} .4 \mathrm{AktG}$ der Gesellschaft auferlegt hat und deren Belastung durch Ausgaben,exzesse“ jedenfalls unter Rückgriff auf die Treuepflicht des Aktionärs gegenüber der Gesellschaft unterbunden werden können. ${ }^{87}$

Die Benennung der Anmeldestelle vom Einverständnis der Gesellschaft unter Hinweis darauf abhängig zu machen, dass der Aktionär deren Einverständnis gegebenenfalls mittels einer einstweiligen Verfügung durchzusetzen vermag, könnte im Übrigen noch aus einen weiteren Grund nicht überzeugen. Zum Erlass einer Sicherungsverfügung gemäß $\ 935 \mathrm{ZPO}$ bedürfte es nämlich zunächst eines entsprechenden Verfügungsanspruchs des ermächtigten Aktionärs, ${ }^{88}$ für den aber keine Rechtsgrundlage ersichtlich ist. Zudem müsste im Vagen bleiben, nach welchen Kriterien das Bestehen eines solchen Anspruchs (?) des ermächtigten Aktionärs auf Zustimmung zu seinen Personalvorschlägen zu beurteilen wäre. In Betracht käme daher allenfalls der Erlass einer Regelungsverfügung nach $\$ 940 \mathrm{ZPO},{ }^{89}$ die nach der obergerichtlichen Rechtsprechung freilich ebenfalls zur Voraussetzung hat, dass bestimmte Ansprüche aus dem streitigen Rechtsverhältnis zur Gesellschaft zumindest entstehen können müssen. ${ }^{90}$

Nach alledem würde mit dem Erfordernis der Zustimmung der Gesellschaft zur Benennung einer Anmeldestelle die überaus bedenkenswerte Maxime verfehlt, dass man „die in der Natur der Sache liegende Antagonie zwischen Vorstand und Aktionärsminderheit gerade für den Fall, dass der Vorstand die Einberufung im Vorfeld abgelehnt hat, nicht noch zusätzlich dadurch schüren [sollte], dass man Kooperation verlangt, wo es ihrer nicht einmal bedarf bzw. eine andere Lösung praktikabler, sicherer und einfacher ist" ${ }^{91}$ Eine solche Lösung steht mit der Anerkennung einer alleinigen Befugnis der ermächtigten und über ein ausgeprägtes Interesse an Durchführung der

86 S. unter II. 2. d).

87 S. oben unter II. 2. f).

88 Statt vieler Reichold, in: Thomas/Putzo, ZPO, 33. Aufl., 2012, $\$ 935$ Rdn. 6 f m. w. N.

89 Zur Bedeutung der Regelungsverfügung im Gesellschaftsrecht s. nur VolLKommer, in: Zöller, ZPO, 30. Aufl., 2012, $\$ 940$ Rdn. 8 m. w. N.

90 OLG Koblenz, NJW-RR 1986, 1039; ReIchold, aaO (Fn. 88), $\$ 940$ ZPO Rdn. 2.

91 Reichert/Balke, aaO (Fn. 36), $\$ 4$ Rdn. 60. 
Hauptversammlung verfügenden Aktionärsminderheit zur Benennung der Anmeldestelle nämlich gerade zur Verfügung.

\section{Ergebnis}

Das Verfahren der Anmeldung zur Hauptversammlung und des Nachweises des Anteilsbesitzes bildet nach allem einen integralen Bestandteil der Einberufungskompetenz und soll die Vorbereitung der Hauptversammlung ermöglichen. Im Fall des $\$ 122 \mathrm{Abs} .3 \mathrm{AktG}$ ist es deshalb der einberufenden und für die Vorbereitung der Hauptversammlung verantwortlichen Aktionärsminderheit gestattet, eine Anmeldeadresse und eine Adresse für den Zugang der Anteilsbesitznachweise anzugeben, und zwar auch dergestalt, dass sie die Anmeldung bzw. Übersendung gegenüber einem von ihr beauftragten Hauptversammlungsdienstleister vorgibt. Wie andere Vorschriften des AktG ist auch $\$ 123$ Abs. 2 Satz 2 AktG, soweit er den Zugang der Anmeldung bei der Gesellschaft statuiert, auf den Normalfall der Einberufung nach Maßgabe des $\ 121$ Abs. 2 Satz 1 AktG zugeschnitten; im Fall des $₫ 122$ Abs. 3 AktG bedarf die Vorschrift der erwähnten sinngemäßen Anwendung. 